\title{
HETEROPHOTOCELL BASED ON SUPER-LINE PHOTOVENTIL EFFECT
}

\author{
V.V. Kovalchuk \\ State Environmental University, Odesa, Ukraine \\ e-mail:lslvvvas@ukr.net
}

The paper considers the superliner photovoltaic effect associated with the modification of heterojunctions of the $\mathrm{pCu}_{2} \mathrm{~S}-\mathrm{nSi}$ type by introducing a silicon nanocluster subsystem into it at the junction boundary. The design of a photocell containing two sequentially articulated $p-n$ junctions of counter action, photoactive in different regions of the spectrum, is proposed.

We show that film heterojunctions, which include a nanocluster subsystem, have a difference from the non-clustered version. Heterophotocells without nanocluster subsystem always have sublinear, or, in rare cases, linear lux-ampere characteristics (up to illumination $\sim 510^{4} \mathrm{~lx}$ ), while because of introducing nanocluster centers into the base p-region of the $\mathrm{Cu}_{2} \mathrm{~S}-\mathrm{Si}$ heterojunctions, a significantly higher integral sensitivity is achieved at high illumination of the samples in the mode of a valve photocell.

Keywords: superliner photovoltaic ejection, heterojunction, nanocluster centers.

Received 15.07.2020; Received in revised form 28.08.2020; Accepted 04.09.2020

\section{Introduction}

The transition from an individual atom to a macrostate characterized by the formation of structures such as quasi-molecular clusters, subcolloid and colloidal nanoclusters (NC), which are extensive in their nature, since they successively change their properties down to the properties of the corresponding macro substance [1]. The latter is very important to be taken into account when using quasi-metallic whiskers as an alloying principle for individual materials and heterostructures (HS).

It is well known that metal-cluster centers of very large sizes- up to colloidal particles 1000 angstroms [2] - can be created in the volume of individual crystals by special technological methods. The effect of nanocrystals of different sizes on the optical and some other properties of crystals ("red shift" of the color of the matrix material with enlargement of centers, etc.) was studied theoretically and experimentally [3]. An analogy with the different stages of the photographic process in alkali-haloid materials and semiconductors would also be appropriate here. In addition, in a single-crystal matrix, it is possible to vary the sizes of NC centers rather widely [4].

Taking into account the specifics of the heterojunction under consideration $\left(\mathrm{pCu}_{2} \mathrm{~S}-\mathrm{nSi}\right)$, nanocrystals are introduced into its structure by implantation: the deposition of a cluster pattern of an island structure on a silicon substrate before the deposition of a copper sulfide layer.

By varying the rates of formation of $\mathrm{NC}$ and the temperature of the substrate, we are able to implant cluster centers in the $\mathrm{pCu}_{2} \mathrm{~S}-\mathrm{nSi}$ heterojunction in the form of a nanocluster subsystem (NCS) of silicon. Thus, a $\mathrm{pCu}_{2} \mathrm{~S}-(\mathrm{Si}-\mathrm{NCS})-\mathrm{nSi}$-type $\mathrm{HS}$ is formed. For such a HS, "extensive" effects are observed, namely spectral-inverted and super linear photovoltaic ones.

It is noted that transition metals ( $\mathrm{Fe}, \mathrm{Ni}, \mathrm{Co}$, etc.) can also be used as an implantable material to create the HS. In this case, the results do not change in any significant way (even when one metal replaces another). The latter is a good confirmation of the fact that the observed effects are due to a greater extent to the presence of NCS, and not to their material. The heterostructure considered in this work contains a silicon NCS. This is due to the advantage of $\mathrm{Si}$ in terms of purity class over the other materials at our disposal.

\section{Superliner photovoltaic effect}

Let us consider the possibility of creating a device with operation based on the revealed superliner photovoltaic effect. From our point of view, it is of interest to comprehensively 
study the main characteristics of samples with NCS for elucidating the mechanisms of transfer in $\mathrm{pCu}_{2} \mathrm{~S}-(\mathrm{Si}-\mathrm{NCS})-\mathrm{nSi}$ HS. Particularly noticeable changes take place in the case of NC with sizes of more than 20 angstroms. Fig. 1 shows the I-V characteristics of the $\mathrm{pCu}_{2} \mathrm{~S}-(\mathrm{Si}-\mathrm{NCS})-\mathrm{nSi} \mathrm{HS}$ with the introduced (according to the formation method we developed and described in [2-4]) cluster raster $\mathrm{Ni}$ (the size of $\mathrm{NC}$ centers is more than 20 angstroms). In Fig. 1 (b) a typical reverse branch of dependence I vs $\mathrm{V}$ for a $\mathrm{pCu}_{2} \mathrm{~S}-$ (NCS)-nSi-type HS is shown. Three sections can be distinguished in it: 1 - prebreakdown area; 2 - area of sharp breakdown; 3 - area of high currents, for which a decrease in the slope of the graph is observed.

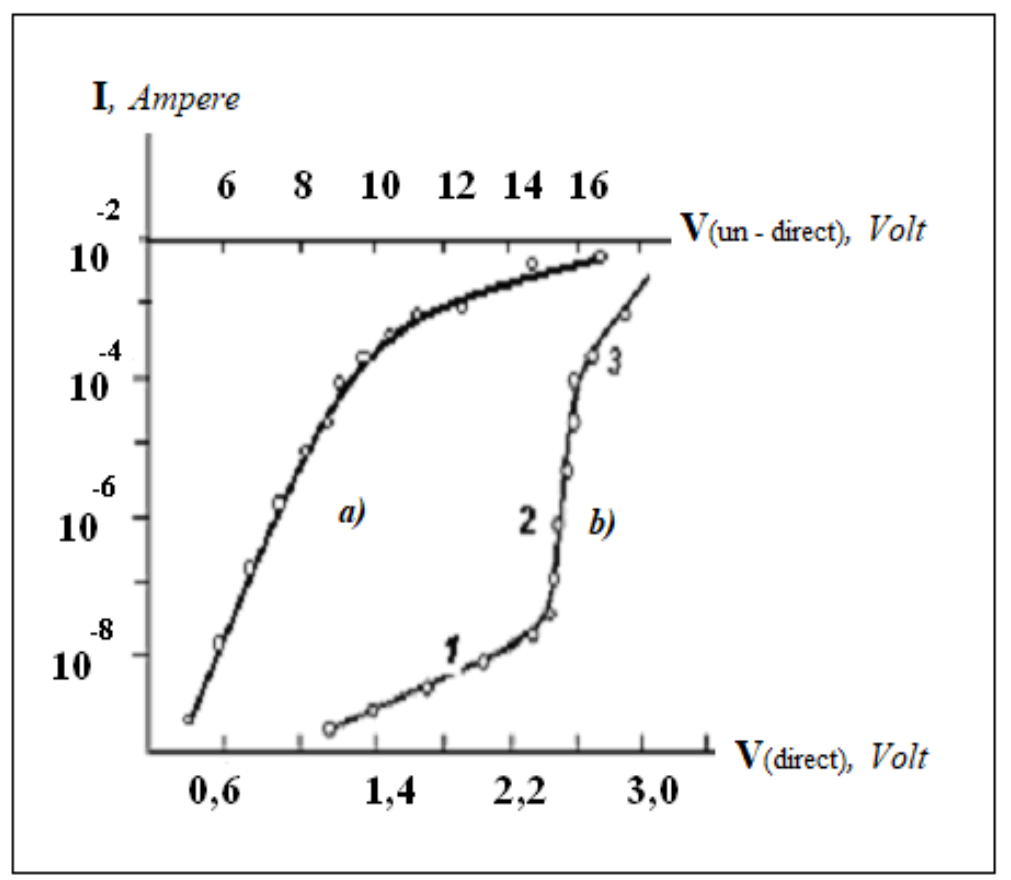

Fig: 1. Typical I vs $\mathrm{V}$ characteristics of the $\mathrm{HS}$ of $\mathrm{pCu} 2 \mathrm{~S}-(\mathrm{Si}-\mathrm{NCS})-\mathrm{nSi}$-type (the size of $\mathrm{NC}$ centers is more than 20 angstroms): $a$ - straight branch; $b$ - reverse branch.

A possible mechanism of carrier multiplication due to NC ionization is illustrated in Fig. 2. In this case, the high quality of the samples is very important; due to it the phenomenon of charge multiplication and the associated avalanche breakdown effect are so clearly manifested in the dependence of I vs V characteristics of the cells. At low voltages, the straight branch of the I vs V characteristic obeys an exponential law ( $\beta \sim 2 \div$ 2.5 ), but with an increase in voltage, the current increases slower than in a HS without NCS.

Changes in the I vs $\mathrm{V}$ characteristics at different temperatures show that the breakdown voltage decreases with decreasing temperature (Fig. 3). This behavior of the I vs $\mathrm{V}$ characteristic is opposite to that observed in the case of the $\mathrm{pCu}_{2} \mathrm{~S}-\mathrm{nSi}$ HS (without NCS) and, as is known, is characteristic of avalanche breakdown of $\mathrm{p}-\mathrm{n}$ junctions.

The comparison of the I vs V characteristics of HS of the $\mathrm{pCu}_{2} \mathrm{~S}-(\mathrm{Si}-\mathrm{NCS})-\mathrm{nSi}$ and $\mathrm{pCu}_{2} \mathrm{~S}-\mathrm{nSi}$ types indicates a slight increase in the first case of the pre-breakdown region currents, which is also explained by the multiplication of current carriers due to the presence of NC forming the NCS of the film HS. 


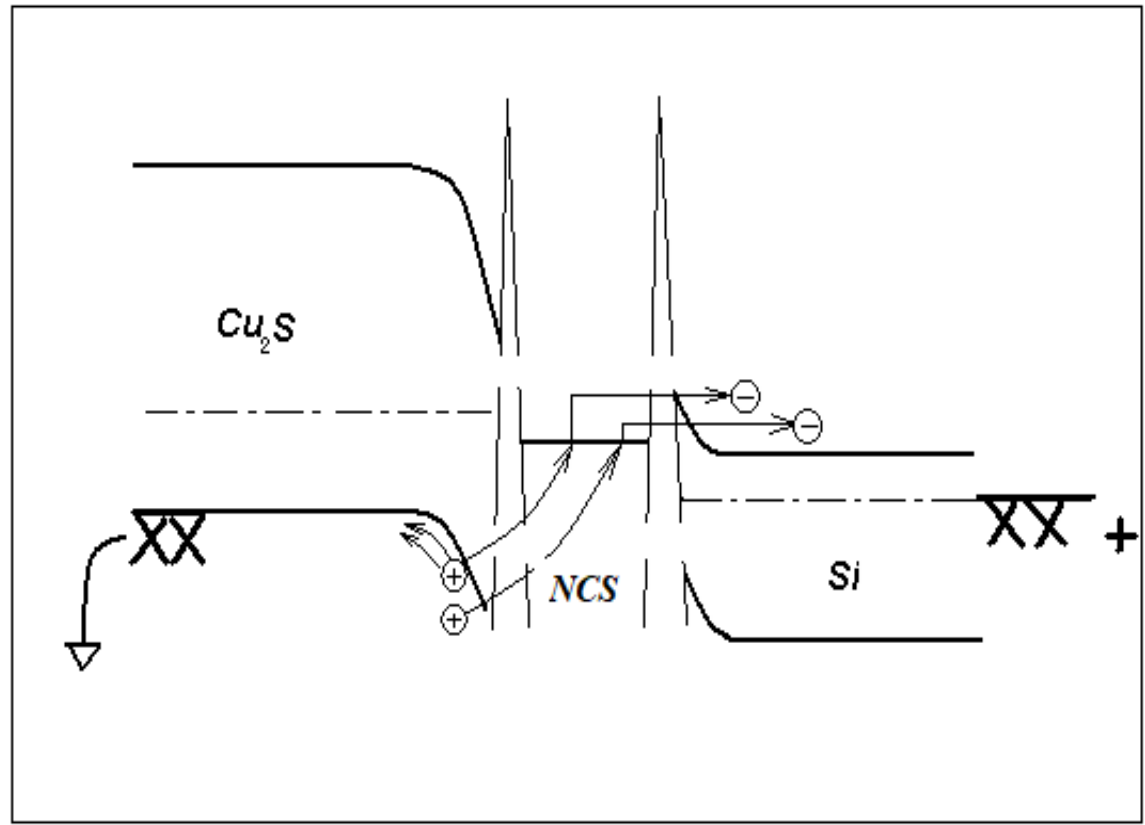

Fig. 2. Illustration of the mechanism of carrier multiplication due to the ionization of NC centers during reverse bias on a $\mathrm{HS}$ of the $\mathrm{pCu}_{2} \mathrm{~S}-(\mathrm{Si}-\mathrm{NCS})-\mathrm{nSi}$ type.

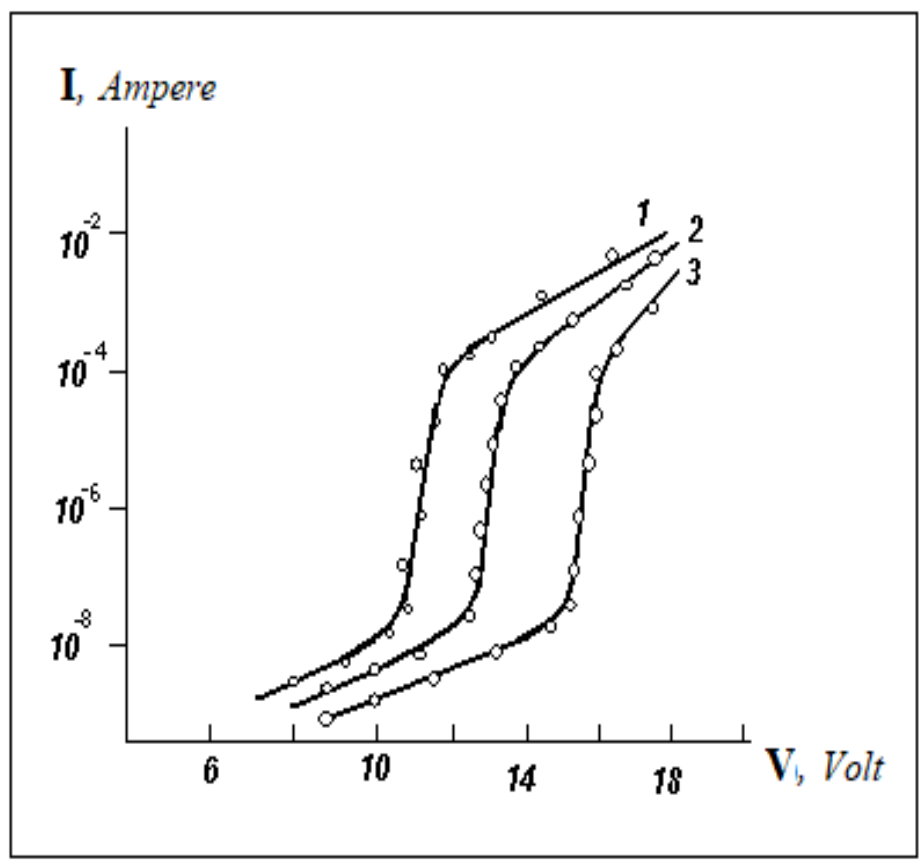

Fig. 3. Volt-ampere characteristics of the $\mathrm{HS}$ of the $\mathrm{pCu}_{2} \mathrm{~S}-(\mathrm{Si}-\mathrm{NCS})-\mathrm{nSi}$ type at temperatures: $1-80 \mathrm{~K} ; 2$ - $150 \mathrm{~K} ; 3-300 \mathrm{~K}$.

An analysis of the photoelectric and capacitance characteristics of $\mathrm{HS}$ of the $\mathrm{pCu}_{2} \mathrm{~S}-$ (Si-NCS)-nSi type confirms the reality of the process of additional carrier creation due to the ionization of $\mathrm{NC}$ centers. At reverse voltages lower than the breakdown 
volt-capacitance characteristics (Fig. 4) of the $\mathrm{pCu}_{2} \mathrm{~S}-(\mathrm{Si}-\mathrm{NCS})-\mathrm{nSi}$-type $\mathrm{HS}$ are described by the dependence $\mathrm{V} \sim \mathrm{C}^{-3}$. Extrapolation of the curve to $\mathrm{C}=0$ indicates an increase in the diffuse potential $\left(\mathrm{V}_{\mathrm{d}} \approx 1.8 \mathrm{~V}\right)$ in comparison with non-clustered transitions. This indicates that, as a result of the introduction of a metal-cluster raster, the potential transition profile becomes more complex (becomes less sharp).

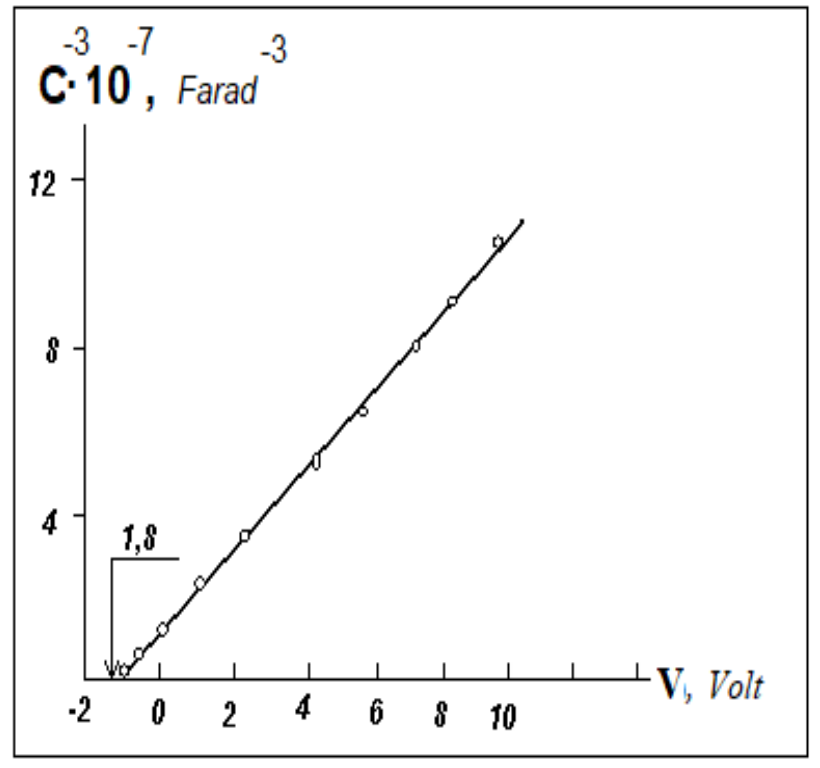

Fig. 4. Capacitance-voltage dependence of the $\mathrm{HS}$ of $\mathrm{pCu}_{2} \mathrm{~S}-(\mathrm{Si}-\mathrm{NCS})-\mathrm{nSi}$ type.

The latter may be due to the total influence of potential barriers associated with the presence of NCS in the structure of the HS, like the way it takes place in the collective barrier model proposed by Prof. M. Sheinkman for a photoconductor with quasimacroscopic inclusions of high-resistance sections [5]. The results obtained indicate that $\mathrm{NCS}$ in the $\mathrm{pCu}_{2} \mathrm{~S}-\mathrm{nSi} \mathrm{HS}$, on the one hand, change the character of the impurity distribution and, on the other hand, significantly increase the potential barrier in the transition region, which is also confirmed in studies of the photoventile effect.

In fact, we discuss the combination of the conventional gate photoeffect in a $\mathrm{p}-\mathrm{n}$ type diode, associated with the barrier separation of non-equilibrium electrons and holes, and the mechanism of photocurrent multiplication due to the interaction of hot photoelectrons with the electron substance of metal cluster centers blocked by asymmetric potential barriers in the HS region. We explain the discovered by us superliner photovoltaic effect in $\mathrm{pCu}_{2} \mathrm{~S}-(\mathrm{Si}-\mathrm{NCS})-\mathrm{nSi}-\mathrm{HS}$, leading to an increase in the photosensitivity of elements at high illumination (Fig. 5), with help of these phenomena.

\section{Manufacturing of a device based on HS in the presence of NCS}

The manufacture of a superliner photocell includes the operation of applying a metal cluster raster of colloidal dispersion (sizes of $\mathrm{NC}$ centers are greater than 20 angstroms) on the surface of a silicon wafer (at $100{ }^{\circ} \mathrm{C}$ ). This procedure is carried out by the thermal evaporation of the corresponding metal (chromium, nickel, iron, or cobalt) in a vacuum $\left(\sim 10^{-5}\right.$ Torr $)$ in a mode that provides a colloidal metal monolayer, clearly resolved with an electron microscope. Moreover, what is important, the sizes of individual particles are from 50 to 100 angstroms, i.e. they are much larger than the $\mathrm{NC}$ centers providing, as 
noted in [3], the spectrally inverted photovoltaic effect. To obtain a raster of NC centers of the indicated sizes, we also successfully used the method of pyroactivation decomposition of the corresponding organometallic compounds.

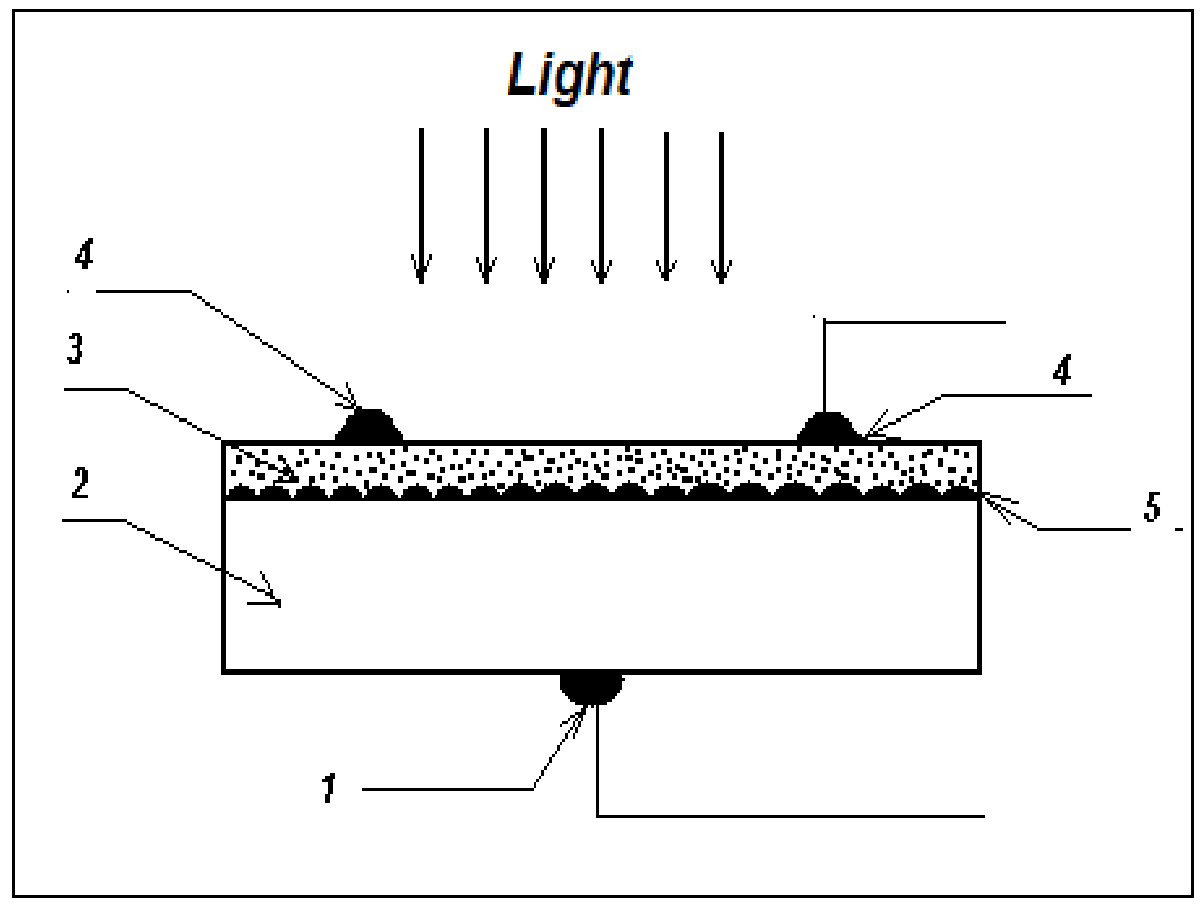

Fig. 5. Schematic representation of a superliner heterophotocell:

1 and 4 - thin-removable contacts; 2 - silicon crystal, 3 - copper sulfide film; 5 - nanocluster raster.

Consider a specific system with $\mathrm{NC}$ centers, i.e. HS of $\mathrm{pCu}_{2} \mathrm{~S}-(\mathrm{Si}-\mathrm{NCS})-\mathrm{nSi}$ type, in contrast to the non-clustered version $\mathrm{pCu}_{2} \mathrm{~S}-\mathrm{nSi}$.

We studied both cases, but as a result, it turns out that $\mathrm{pCu}_{2} \mathrm{~S}-\mathrm{nSi}$-photocells always have a sublinear or, in rare cases, a linear lux-ampere characteristic (up to illumination $\sim 5 \cdot 10^{4} \mathrm{~lx}$ ), while in $\mathrm{pCu}_{2} \mathrm{~S}-(\mathrm{NCS})-\mathrm{nSi}$-photocells at illumination of more than $8 \cdot 10^{3} \mathrm{~lx}$, a superlinear dependence is clearly manifested on the lux-ampere characteristic (Fig. 6).

The values of the slope coefficients of the rectified lux-ampere sections with respect to the abscissa axis, equal to 1 , correspond to a linear dependence, less than 1 - to sublinear, and more than 1 - to superlinear (or superliner) dependence.

In accordance with the above, we will further distinguish between "linear" (L) (more precisely, quasilinear, closer to sublinear) and "superliner" (SL) modes of operation of photocells.

Photocell sample comparison with different options: (a) $\mathrm{pCu}_{2} \mathrm{~S}-\mathrm{nSi}$ and (b) $\mathrm{pCu}_{2} \mathrm{~S}-(\mathrm{Si}-\mathrm{NCS})-\mathrm{nSi}$ shows that at moderate low light intensities, both types of photocells are practically equivalent in their main characteristics. This indicates the fact that in the L-mode of operation $\mathrm{pCu}_{2} \mathrm{~S}-(\mathrm{Si}-\mathrm{NCS})-\mathrm{nSi}$-photocells, quasi-metallic centers behave passively with respect to the photovoltaic effect that can be explained by the presence of a specific Coulomb barrier for these centers, which limited their photoelectric activity and electron-exchange interaction with the conduction and valence bands of the semiconductor. Colloidal aggregates with similar properties are found, for example, in alkali halide crystals and in selenium films treated in mercury vapor. 


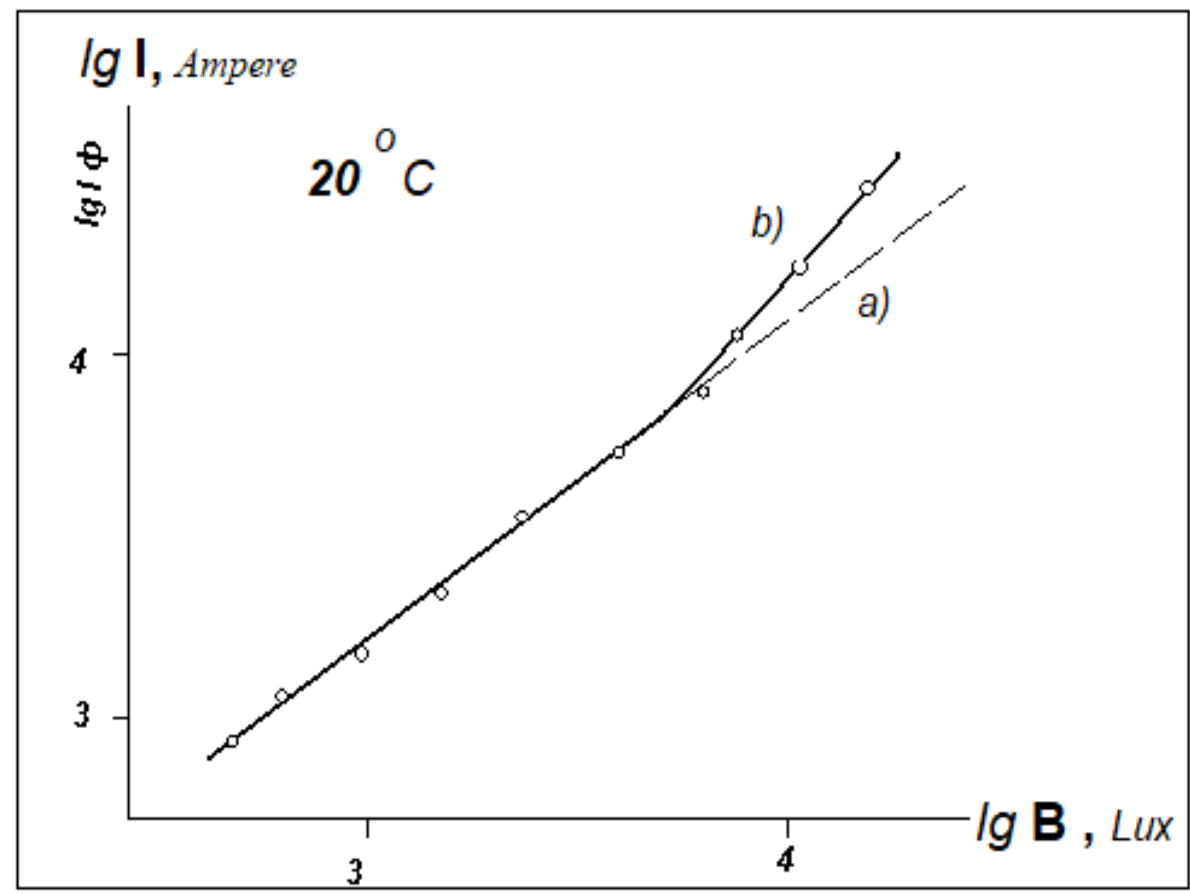

Fig: 6. Lux-ampere characteristics of the HS:

(a) $\mathrm{pCu}_{2} \mathrm{~S}-\mathrm{nSi}$ and (b) $\mathrm{pCu} 2 \mathrm{~S}-(\mathrm{Si}-\mathrm{NCS})-\mathrm{nSi}$.

The linear generation regime $\left(<10^{4} \mathrm{~lx}\right)$ is mainly explained by the band diagram of the proper $\mathrm{pCu}_{2} \mathrm{~S}-\mathrm{nSi} \mathrm{HS}$ plotted from the data of measurements of the capacitance and I vs V characteristics of the HS, as well as the electrical and optical properties of the base regions.

A significant difference appears in $\mathrm{pCu}_{2} \mathrm{~S}-(\mathrm{Si}-\mathrm{NCS})-\mathrm{nSi}$ photocells in the area of high illumination when the mechanism converting them to the superliner operating mode (Fig. 6, b) is triggered. The scheme proposed by us for switching between linear and superliner modes, taking into account the specific structure of the sample, is reduced to the following. At high light intensities $\left(\sim 10^{4} \mathrm{Lx}\right)$, the conductivity of the photosensitive $\mathrm{Cu}_{2} \mathrm{~S}$ film significantly increases (by 1-2 orders of magnitude), the Debye shielding length decreases, and the colloidal center is solvated by mobile carriers (Fig. 7).

As a result, NC centers begin to participate in electron exchange with the conduction and valence bands. Further, the specificity of the system lies in the fact that the electrons entering the $\mathrm{NC}$ centers from $\mathrm{Cu}_{2} \mathrm{~S}$ are "hot" (Fig. 7b).

Excess energy, as in a massive metal, is lost either to dissipation in the quasilattice of $\mathrm{NC}$ centers or on collisions with electrons. Assuming that Maxwellization is more effective than cooling, we end up with heating of the electron "vapor" in the NC centers. The latter, in turn, leads to the fact that the secondary emission of electrons at $\mathrm{NC}$ centers can exceed the intensity of the primary exciting electron flux, i.e. an additional current-generating mechanism is involved in this case. The effect is enhanced by the fact that the electron temperature is included in the exponential Boltzmann factor $\exp (-\Delta E / k T)$, which determines the probability of electron ejection from the quasi-metallic center.

By introducing $\mathrm{NC}$ centers into the base p-region of the $\mathrm{Cu}_{2} \mathrm{SXSi}$-type $\mathrm{HS}$, a significantly higher integral sensitivity is achieved at high illumination of the 66 
samples (more than $8 \cdot 10^{3} 1 \mathrm{x}$ ) in the valve photocell mode: $2.4 \mathrm{~mA} / \mathrm{lx}$ versus 1.8 $\mathrm{mA} / \mathrm{lx}$ in the usual way.

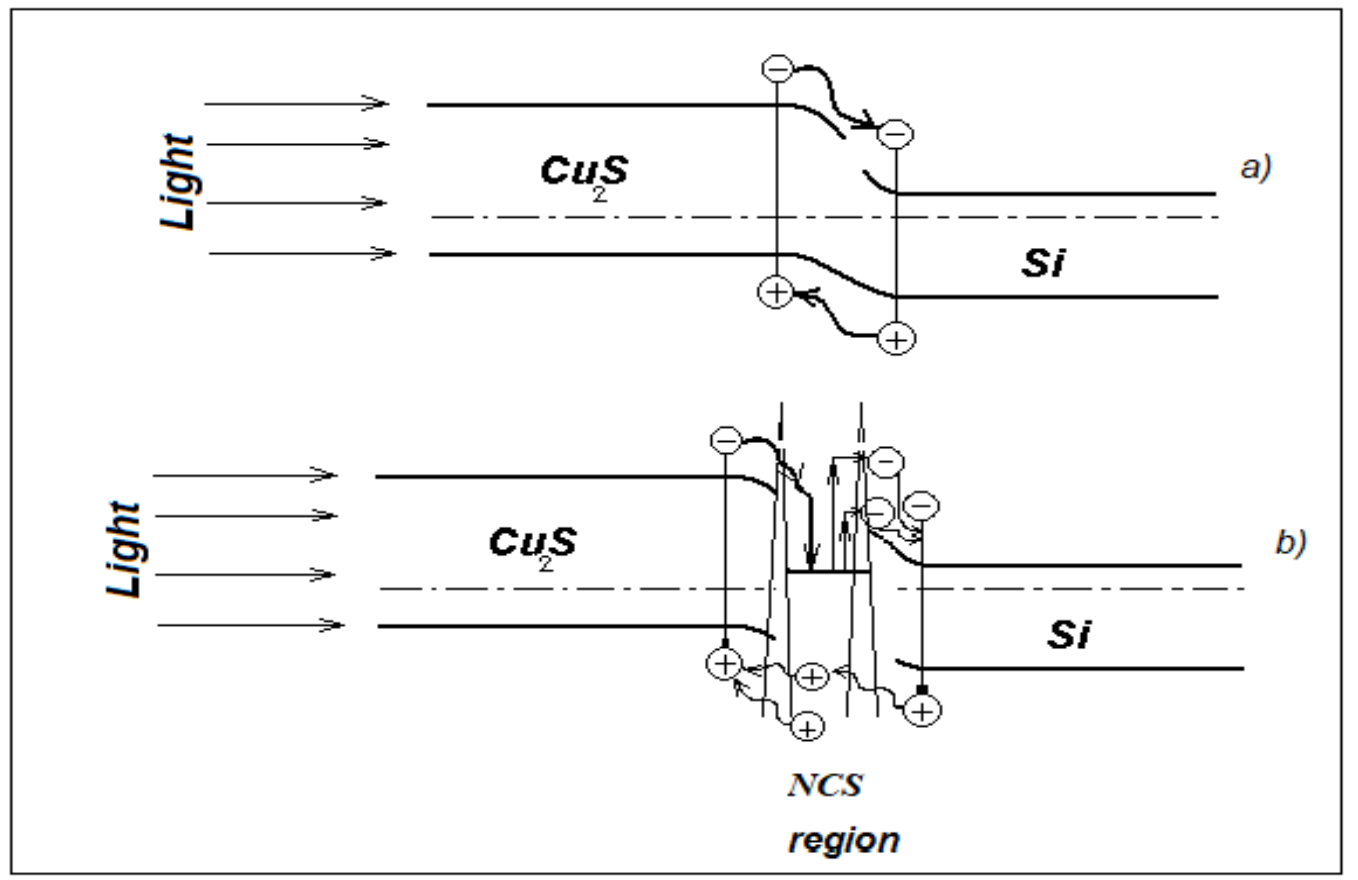

Fig. 7. Zone energy diagrams of HS:

(a) $\mathrm{pCu}_{2} \mathrm{~S}-\mathrm{nSi}$ and (b) $\mathrm{pCu} 2 \mathrm{~S}-(\mathrm{Si}-\mathrm{NCS})-\mathrm{nSi}$.

\section{Discussion and conclusion}

The paper presents the results of a study of the $\mathrm{pCu}_{2} \mathrm{~S}-\mathrm{nSi}$ heterojunction (HJ), which deserves attention on the way to solving the problems of modern functional diagnostics of devices with NCS, as well as in the development of photoelements (PE). The proposed technology for obtaining $\mathrm{HJ}$ is based on the formation of clustered phases in the form of NC centers. This is evidenced by electron diffraction patterns and photomicrographs of a film portion of silicon nanoparticles and a NCS based on it [2-4]. The optimal operating modes of a heterophotocell based on NC of the $\mathrm{pCu}_{2} \mathrm{~S}-(\mathrm{Si}-\mathrm{NCS})-$ nSi type are determined.

The technology for preparing $\mathrm{HJ}$ of the $\mathrm{pCu}_{2} \mathrm{~S}-(\mathrm{Si}-\mathrm{NCS})-\mathrm{nSi}$ type provides a predominantly smooth band-energy profile of the transition, which allows to use this structure as a basis for photo energy converters. Modification of the properties of HJ of the $\mathrm{pCu}_{2} \mathrm{~S}-\mathrm{nSi}$ type is realized by introducing an NCS. The change in the structure of the $\mathrm{HJ}$ of $\mathrm{pCu}_{2} \mathrm{~S}-\mathrm{nSi}$ type can occur through the process of implantation of the cluster pattern of island structure on a silicon substrate before the deposition of a copper sulfide layer. Varying the rate of formation of $\mathrm{NC}$ centers and the temperature of the substrate opens the possibility of implanting NC centers with geometric dimensions from 10 to 150 angstroms.

We note that not only the spectrally inverted effect [3], but also the superliner photoeffect is observed on a HS of this type. Such processes are extensive in their nature since they are largely determined by the geometry and morphology of $\mathrm{NC}$ centers, and not by their material. 
At an increase in the size of NC centers to hundreds and more angstroms, the photoeffect is not only not enhanced, but, on the contrary, completely disappears, giving way to a new effect of superlinearity of the lux-ampere characteristic. The discovered superliner photoeffect in a heterophotocell based on an NCS leads to an increase in the cell photosensitivity at high illumination. The manufacture of a superliner photocell includes the operation of applying a metal cluster raster of colloidal dispersion (with NC centers larger than 20 angstroms) on the surface of a silicon wafer (at $100{ }^{\circ} \mathrm{C}$ ). We show that film HJ, which include NCS, demonstrate a difference from the non-clustered version. Heterophotocells without NCS always have sublinear or, in rare cases, linear luxampere characteristics (up to illumination $\sim 510^{4} \mathrm{~lx}$ ), while as a result of introducing $\mathrm{NC}$ centers into the base p-region of the $\mathrm{Cu}_{2} \mathrm{~S}-\mathrm{Si} \mathrm{HS}$, a significantly higher integral sensitivity is achieved at high illumination of the samples in the mode of a valve photocell.

\section{References}

1. Sattler, K. Cluster assembled materials / K. Sattler // Zurich: Trans. Tech. Publ 1996. - Vol. 232. - P. $12-87$.

2. Kovalchuk, V. Cluster modification of semiconductor hetero structures / V. Kovalchuk // K.: Hi-Tech., 2007. - 309 p.

3. Kovalchuk, V. Photo receiver with nanocluster subsystem / V. Kovalchuk // Technium: Romanian Journal of Applied Sciences and Technology. - 2020. - Vol. 2 (3). - P. $62-67$.

4. Kovalchuk, V. Optical properties of clusters / V. Kovalchuk // J. of Physics \& Electronics. - 2018. - Vol. 26 (1). - P. $29-34$.

5. Sheinkman, M. Svoystva I mekhanizmy ostatochnoy provodimosti V monokristallakh CdS:Ag:Cl / M. Sheinkman, I. Markevich // Fizika tverdogo tela. 1970. - Volume 12 (11). - P. 3130 - 3140. 